\title{
Early echocardiographic findings in patients hospitalized for COVID-19 pneumonia: a prospective, single center study
}

\author{
Elisa Ceriani ${ }^{1}\left(10\right.$ ) Azzurra Marceca ${ }^{2} \cdot$ Antonio Lanfranchi $^{2} \cdot$ Stefano De Vita $^{3} \cdot$ Riccardo Schiavon $^{4}$. \\ Francesco Casella ${ }^{4}$. Daniela Torzillo ${ }^{4} \cdot$ Marta del Medico $^{5} \cdot$ Diego Ruggiero $^{6} \cdot$ Alberto Barosi $^{3} \cdot$ Chiara Cogliati $^{4}$
}

Received: 13 January 2021 / Accepted: 30 March 2021 / Published online: 21 May 2021

(c) Società Italiana di Medicina Interna (SIMI) 2021

\begin{abstract}
Background Cardiac dysfunction, mainly assessed by biomarker alterations, has been described in COVID-19 infection. However, there are still areas of uncertainty regarding its effective role in disease evolution. Aim of this study was to evaluate early echocardiographic parameters in COVID pneumonia and their association with severity disease and prognosis. Methods An echocardiographic examination was performed within $72 \mathrm{~h}$ from admission in 64 consecutive patients hospitalized for COVID-19 pneumonia in our medium-intensity care unit, from March 30th to May 15th 2020. Six patients were excluded for inadequate acoustic window.

Results Fifty-eight consecutive patients were finally enrolled, with a median age of 58 years. Twenty-two (38\%) were classifiable as severe COVID-19 disease. Eight out of 58 patients experienced adverse evolution (six died, two were admitted to ICU and received mechanical ventilation), all of them in the severe pneumonia group. Severe pneumonia patients showed higher troponin, IL-6 and D-Dimer values. No significant new onset alterations of left and right ventricular systolic function parameters were observed. Patients with severe pneumonia showed higher mean estimated systolic pulmonary artery pressure (sPAP) $(30.7 \pm 5.2 \mathrm{mmHg}$ vs $26.2 \pm 4.3 \mathrm{mmHg}, p=0.006)$, even if in the normality range values. No differences in echocardiographic parameters were retrieved in patients with adverse events with respect to those with favorable clinical course. Conclusion A mild sPAP increase in severe pneumonia patients with respect to those with milder disease was the only significant finding at early echocardiographic examination, without other signs of new onset major cardiac dysfunction. Future studies are needed to deepen the knowledge regarding minor cardiac functional perturbation in the evolution of a complex systemic disorder, in which the respiratory involvement appears as the main character, at least in non-ICU patients.
\end{abstract}

Keywords Echocardiography $\cdot$ COVID-19 $\cdot$ Cardiac dysfunction $\cdot$ Systolic pulmonary artery pressure

Elisa Ceriani

elisa.ceriani@live.com

1 Department of Internal Medicine, L. Sacco Hospital, University of Milan, Via GB Grassi, 20157 Milan, Italy

2 Cardiovascular Imaging Unit, Department of Cardiology, L. Sacco Hospital, University of Milan, Milan, Italy

3 Cardiovascular Imaging Unit, Department of Cardiology, L. Sacco Hospital, University of Milan, Milan, Italy

4 Department of Internal Medicine, L. Sacco Hospital, University of Milan, Milan, Italy

5 Department of Internal Medicine, L. Sacco Hospital, University of Milan, Milan, Italy

6 Electrophysiology Unit, Department of Cardiology, L. Sacco Hospital, University of Milan, Milan, Italy

\section{Background}

The global pandemic of the novel coronavirus disease 2019 (COVID-19) caused by severe acute respiratory syndrome coronavirus 2 (SARS-CoV-2) began in China in December 2019 and spread globally a few months later [1].

Although it seems to spare the cardiovascular system as the primary site of infection, there is increasing evidence linking COVID-19 with cardiovascular morbidity [2]. A discrete prevalence of cardiac damage, defined by troponin elevation independently from electrocardiographic and echocardiographic findings, has been reported in patients hospitalized for COVID infection, with significant correlation with mortality [3-5]. Several pathways have been proposed for cardiovascular involvement during COVID illness. Firstly, direct heart involvement has been described, with a 
clinical picture of myopericarditis [6,7]. Moreover, cardiac damage could be mediated by systemic cytokinine toxicity during the hyperinflammation stage, inducing an increase in troponin values [4, 8]. However, troponin elevation in COVID-19 patients could represent the consequence of a more severe systemic disease, not necessarily being indicative of primary cardiac dysfunction [4, 9]. Finally, the strict heart-lung interaction should be considered, in particular in the setting of the most severe cases of COVID pneumonia, where a right ventricle (RV) dysfunction has been reported [10]. This condition is mainly described in ICU patients with advanced stages of the disease, when substantial alterations of heart-lung interaction have already occurred, both due to disease itself and to mechanical ventilation consequences.

Although there is increasing evidence of cardiovascular involvement during COVID disease, there are still areas of uncertainty regarding its effective role in determining disease evolution, in particular in the early phases of the disease course. In this regard, the aim of this study was to evaluate the eventual presence of early cardiac dysfunction and its prognostic role by means of echocardiography in consecutive COVID pneumonia patients, hospitalized in a mediumintensity care department.

\section{Methods}

\section{Study design and participants}

We performed a prospective study at the Internal Medicine Department of Luigi Sacco University Hospital (Milan, Italy) from March 30th to May 15th, 2020. Our Institution is one of the major regional tertiary teaching hospitals that managed COVID-19 patients, and the Milan Hub for Infectious Diseases. The Internal Medicine Department was commuted into a COVID-19 medium-intensity care unit for the whole duration of the emergency [11]. The level of intensity of care of this department ranged from low-flow oxygen delivery to non-invasive ventilation. All adult patients $(>18$ years old) admitted to our medical ward for COVID pneumonia were considered eligible. The following conditions were considered exclusion criteria: pregnancy, absence of consent to participate in the study or a non-interpretable echocardiographic examination as defined below.

The study protocol complied with the Declaration of Helsinki. The study was approved by the local Ethics Committee and informed consent was obtained.

\section{Data collection}

All patients enrolled in the study were admitted from the Emergency Department (ED) to the ward with a diagnosis of COVID pneumonia based on a positive nasopharyngeal swab and the presence of pulmonary infiltrate at chest X-ray or computed tomography scan. Accurate past medical history was obtained together with data about pre-existing cardiovascular morbidity and ongoing treatment. Previous echocardiographic data were registered if available. Time from symptoms onset was also examined.

All patients received the following tests at admission: arterial blood gas analysis, 12 leads EKG, complete blood panel including high sensitivity Troponin $\mathrm{T}$ (hs-TnT), D-Dimer and Interleukin-6 (IL-6). Values were considered normal if the following were met: hs-TnT $\leq 15 \mathrm{mg} / \mathrm{L}$, D-Dimer $<500 \mathrm{mcg} / \mathrm{L}, \mathrm{IL}-6<7 \mathrm{ng} / \mathrm{L}$.

The partial arterial oxygen pressure to fractional inspired oxygen ratio $(\mathrm{P} / \mathrm{F})$ was calculated for each patient.

Adverse outcomes were death and/or intubation during hospitalization.

\section{Echocardiographic examination and image interpretation}

The echocardiographic examination was performed within $72 \mathrm{~h}$ from admission. If the patient was on CPAP ventilation therapy, the echocardiographic examination was performed during brief $30 \mathrm{~min}$ "CPAP free" windows, when standard oxygen therapy (through high flow cannula, reservoir or Venturi mask) was administered. This protocol allowed to avoid the effect of positive end expiratory pressure itself on right ventricle function and pressure. Examinations were performed at bedside using a Philips CX-50 portable device by operators (FC; EC, MD, AL; DT) with minimum level-2 echocardiography competence (proficiency defined according to ACC/ASE societies) [12]. Each examination was subsequently interpreted off-line by level-3 echocardiography competence cardiologists (SD, AM) blinded to clinical information, using TomTec ${ }^{\circledR}$ analysis software. The examination interpretation was further supervised by a third experienced echocardiographist $(\mathrm{AB})$ to promote measurement standardization. The echocardiographic examination was performed by operators protected by suitable personal protective equipment [13]. COVID-19 infections in operators were regularly assessed on a clinical basis; at the end of the study period serology was performed in all operators involved in the image acquisition.

Measures were defined according to the latest European and American Echocardiography Society guidelines [14, 15]. Briefly, left ventricle (LV) function was considered normal if the ejection fraction (EF) was $>52 \%$ for males and $>54 \%$ for females. Ejection fraction was evaluated by the Simpson biplane method. Left ventricle mass was estimated by the linear method; relative wall thickness was also calculated. For right ventricular (RV) function the adopted normal cutoff values were: fractional area change (FAC) $>35 \%$; Tricuspid Annular Plane Systolic Excursion 
(TAPSE) $>17 \mathrm{~mm}$, Tissue Doppler-derived right ventricular systolic excursion velocity $\left(\mathrm{S}^{\prime}\right)>9.5 \mathrm{~cm} / \mathrm{s}$. Systolic Pulmonary Artery Pressure (sPAP) was calculated adding right atrioventricular gradient (estimated by tricuspid regurgitation continuous wave Doppler) and central venous pressure (estimated from inferior vena cava expiratory diameter and collapsibility index), with normal value $\leq 35 \mathrm{mmHg}$. The inferior vena cava collapsibility index was evaluated with a sniff, as suggested by guidelines [15]. Pulse wave Doppler was used to measure Pulmonary Artery Acceleration Time (pACT, normal value $<105 \mathrm{~ms}$ ). Finally, the presence or absence of pericardial effusion was reported.

\section{Aim of the study and statistical analysis}

The primary endpoint was to describe echocardiographic characteristics of our cohort of patients hospitalized for COVID pneumonia. Two subgroups of patients will be considered for a better disease characterization: those patients with mild to moderate respiratory involvement and those classifiable with severe disease according to the NIH classification for COVID disease, relying on respiratory failure severity [16].

The secondary endpoint was to evaluate the impact of echocardiographic findings, clinical and laboratory variables on prognosis comparing two groups of patients, those with and without adverse outcomes.

The following correlations were made: laboratory parameters (Hs-TnT, D-Dimer, IL-6) with main LV and RV functional parameters.

Data were expressed as percentages, mean and standard deviations (SD), or median with interquartile range (IQR) for skewed distributions. Independent Student $T$-test was used to compare continuous variables with normal distribution, while the Mann-Whitney test was used for variables with non-normal distribution. Categorical data were assessed among groups by the $\chi^{2}$ test or the Fisher exact test, as appropriate.

\section{Results}

\section{Demographic and clinical characteristics}

Sixty-four consecutive patients diagnosed as having COVID19 pneumonia were eligible for the study. Six patients were excluded because of a poor acoustic window. The demographic and clinical characteristics are summarized in Table 1.

The median age was 58.5 years (IQR 46-75) and 21 patients $(36 \%)$ were women. Thirty patients $(52 \%)$ had one or more comorbidities. Among the comorbidities, the most represented were hypertension in 21 patients (36\%) and diabetes mellitus in eight patients (13\%).

The median duration of symptoms before hospital admission was 6 days (IQR 3-10). The median time from admission to discharge or event occurrence was 9 days (IQR 6-15.5). Twenty-two of 58 patients (38\%) were attributed to the severe pneumonia group, 15 of them were treated with Continuous Positive Airway Pressure (CPAP) with a PEEP range of $7.5-12 \mathrm{cmH} 2 \mathrm{O}$.

Compared with those with milder disease, the severely affected patients were older [(68 years (IQR 57-78.3) vs 51.5 years (IQR $40-70.5$ ), $p=0.006$ ], while no significant differences were observed in gender and comorbidities, except for diabetes mellitus, that was more frequent in the severe pneumonia subgroup [6/22 (27\%) vs $2 / 36(6 \%)$; $p=0.044)]$.

Pathological EKG findings were documented in eight out of 57 patients (14\%), with no significant differences in the two severity subgroups of patients and in those who reached the composite outcome. Among patients with EKG alterations, only one received a diagnosis of unstable angina upon further evaluation, due to stressor-related exacerbations of an already known cardiac ischemic disease.

Three patients were diagnosed as having non-massive pulmonary embolism, two of them with severe respiratory failure accordingly to NIH classification. All of them survived.

Eight out of 58 (14\%) patients reached the composite outcome (six died, two were admitted to ICU and received mechanical ventilation), all of them in the severe pneumonia group.

The patients who reached the composite event were older [77.5 years (IQR 66.3-82) vs 57 years (IQR 43-72), $p=0.005]$, with no significant differences in comorbidities except for hypertension, which was more frequent in the adverse outcome group [6/8 (75\%) vs 15/50 (30\%), $p=0.015]$.

\section{Laboratory results}

Cardiac troponin T and D-Dimer were elevated in 19 (33\%) and in $32(56 \%)$ patients respectively. Median IL-6 values were 25 (9.5-97.0) ng/L. Hs-TnT positivity was found in 12 out of $20(62 \%)$ of severe pneumonia patients vs seven out of $34(21 \%)$ of non-severe patients, $p: 0.003$.

D-Dimer positivity was found in 17 out of $22(77 \%)$ of severe pneumonia patients vs 15 out of 35 (45\%) in nonsevere patients, $p=0.011$. Of notice, we performed compressive ultrasound of inferior leg proximal veins in all patients, as part of local practice for COVID-19 in-patients, and we found one deep vein thrombosis case. This patient belonged to the severe pneumonia group and showed a D-Dimer value $>500$. 
Table 1 Clinical and laboratory characteristics in the study population, in the subgroups of severe vs non-severe pneumonia and in patients who experienced adverse outcome during hospitalization vs patients who didn't experienced adverse events

\begin{tabular}{|c|c|c|c|c|c|c|c|}
\hline \multirow{2}{*}{$\begin{array}{l}\text { Clinical and labora- } \\
\text { tory characteristics }\end{array}$} & \multirow{2}{*}{$\begin{array}{l}\text { All patients } \\
(n=58)\end{array}$} & \multicolumn{3}{|l|}{ Severe pneumonia } & \multicolumn{3}{|l|}{ Adverse outcome } \\
\hline & & No $(n=36)$ & Yes $(n=22)$ & $p$ value & No $(n=50)$ & Yes $(n=8)$ & $p$ value \\
\hline Age. years ${ }^{a}$ & $58.5(46-75)$ & $51.5(40-70.5)$ & $68(57-78.25)$ & 0.006 & $57.0(43-72)$ & $77.5(66.25-82)$ & 0.005 \\
\hline Female sex, $n(\%)$ & $21(36)$ & $14(39)$ & $7(32)$ & 0.587 & $17(34)$ & $4(50)$ & 0.443 \\
\hline $\begin{array}{l}\text { Arterial hypertension, } \\
n(\%)\end{array}$ & $21(36)$ & $10(28)$ & $11(50)$ & 0.088 & $15(30)$ & $6(75)$ & 0.021 \\
\hline $\begin{array}{r}\text { Coronary artery } \\
\text { disease, } n(\%)\end{array}$ & $2(3)$ & $0(0)$ & $2(9)$ & 0.140 & $1(2)$ & $1(12)$ & 0.259 \\
\hline Heart failure, $n(\%)$ & $3(5)$ & $2(6)$ & $1(4)$ & 1.000 & $3(6)$ & $0(0)$ & 1.000 \\
\hline $\begin{array}{l}\text { Chronic obstructive } \\
\text { lung disease, } n(\%)\end{array}$ & $3(5)$ & $2(6)$ & $1(4)$ & 1.000 & $3(6)$ & $0(0)$ & 1.000 \\
\hline $\begin{array}{l}\text { Diabetes Mellitus, } \\
n(\%)\end{array}$ & $8(13)$ & $2(6)$ & $6(27)$ & 0.044 & $7(14)$ & $1(12)$ & 1.000 \\
\hline $\begin{array}{l}\text { Atrial fibrillation, } n \\
(\%)\end{array}$ & $6(10)$ & $2(6)$ & $4(18)$ & 0.187 & $4(8)$ & $2(25)$ & 0.189 \\
\hline $\begin{array}{l}\text { ECG alterations, } n \\
(\%)\end{array}$ & $8 / 57(14)$ & $5 / 36(14)$ & $3 / 21(14)$ & 1.000 & $7 / 50(14)$ & 1/7 (14) & 1.000 \\
\hline Troponin $\mathrm{T}(\mathrm{ng} / \mathrm{L})^{\mathrm{a}}$ & $8.5(5.75-21.5)$ & $6.5(4.75-11.75)$ & $17.5(10.25-41.5)$ & $<0.001$ & $7.0(5.0-16.0)$ & $21(16.5-38.5)$ & 0.002 \\
\hline $\begin{array}{l}\text { Troponin T positivity } \\
(>15 \mathrm{ng} / \mathrm{L})\end{array}$ & $19 / 54(33)$ & $7 / 34(21)$ & $12 / 20(60)$ & 0.003 & $12 / 46$ & 7/8 (87) & 0.002 \\
\hline $\mathrm{D}-\operatorname{Dimer}(\mathrm{mcg} / \mathrm{L})^{\mathrm{a}}$ & $630(367.5-1262.0)$ & $444(327-851)$ & $1152(582-3646)$ & 0.002 & $441(348-851)$ & $\begin{array}{c}3940(1167.75- \\
17,450.75)\end{array}$ & 0.001 \\
\hline $\begin{array}{l}\text { D-dimer positivity } \\
(>500 \mathrm{mcg} / \mathrm{L})\end{array}$ & $32 / 57(56)$ & $15 / 35(43)$ & $17 / 22(77)$ & 0.011 & $24 / 49(49)$ & $8 / 8(100)$ & 0.007 \\
\hline Interleukin-6 (ng/L) ${ }^{\mathrm{a}}$ & $25(9.5-97.0)$ & $14(5.25-29.0)$ & $116(37.5-247.5)$ & $<0.001$ & $18(7.0-46.0)$ & $237(153.0-354.0)$ & $<0.001$ \\
\hline $\mathrm{P} / \mathrm{F}^{\mathrm{b}}$ & $305 \pm 142$ & $402.91 \pm 75.73$ & $153.86 \pm 68.08$ & $<0.001$ & $340.04 \pm 121.38$ & $95.25 \pm 40.90$ & $<0.001$ \\
\hline CPAP, $n(\%)$ & $15(26)$ & $0(0)$ & $15(68)$ & $<0.001$ & $9(18)$ & $6(75)$ & 0.003 \\
\hline
\end{tabular}

${ }^{\mathrm{a}}$ Data reported as median (IQR)

${ }^{\mathrm{b}}$ Data reported as mean $\pm \mathrm{SD}$

Median IL-6 values were higher in the more severely affected group of patients: 116 (IQR 7.5-247.5) ng/L vs 14 (IQR 5.25-29.0) ng/L, $p<0.001$.

Similar laboratory differences observed in severe vs nonsevere pneumonia patients were observed when patients who experienced an adverse outcome were compared with those with an uneventful clinical course (Table 1).

\section{Echocardiographic characteristics}

The echocardiographic characteristics of the population are summarized in Table 2. Median time from admission to echocardiographic examination was 2 days (IQR 1-2). In non-invasively ventilated patients, time range between CPAP support interruption and echocardiography performance was 10-15 min.

Considering the entire population as a whole, no significant alterations of right ventricular systolic function parameters were observed, with measured values of tricuspid annulus peak systolic excursion (TAPSE), RV fractional area change (FAC) and RV S' falling within the normal range of healthy subjects. The same findings were seen when evaluating the right ventricular basal diameter and the right ventricle/left ventricle ratio. The only patient presenting with right ventricular systolic dysfunction had a pre-existing chronic cor pulmonale. Of notice, patients with pulmonary embolism presented normal echocardiographic findings.

When comparing the patients presenting with severe pneumonia, mean estimated Pulmonary Artery Systolic Pressure (sPAP) values resulted higher in severely affected patients $(30.7 \pm 5.2 \mathrm{mmHg}$ vs $26.2 \pm 4.3 \mathrm{mmHg}$ respectively, $p=0.006$ ) (Table 2).

Reduced left ventricular EF was identified in four patients; in three of them this condition was pre-existing, while in one patient it was related to the acute coronary syndrome. All these patients belonged to the non-severe pneumonia group. No significant differences in left side chamber dimensions and function were identified when comparing the severe vs non-severe pneumonia patients or those that reached the composite event vs those with an uneventful clinical course. Notably, relative wall thickness was significantly higher in severe pneumonia patients with a tendency 
Table 2 Echocardiographic characteristics in the study population, in the subgroups of severe vs non-severe pneumonia and in patients who experienced adverse outcome during hospitalization vs patients who didn't experienced adverse events

\begin{tabular}{|c|c|c|c|c|c|c|c|}
\hline \multirow[t]{2}{*}{ Echocardiographic characteristics } & \multirow{2}{*}{$\begin{array}{l}\text { All patients } \\
(n=58)\end{array}$} & \multicolumn{3}{|c|}{ Severe pneumonia } & \multicolumn{3}{|c|}{ Adverse outcome } \\
\hline & & No $(n=36)$ & Yes $(n=22)$ & $p$ value & No $(n=50)$ & Yes $(n=8)$ & $p$ value \\
\hline Left atrial indexed volume $\left(\mathrm{mL} / \mathrm{m}^{2}\right)^{\mathrm{a}}$ & $32.7 \pm 13.7$ & $32.6 \pm 13.2$ & $33.1 \pm 14.8$ & 0.887 & $33.9 \pm 13.9$ & $25.1 \pm 9.5$ & 0.114 \\
\hline $\begin{array}{l}\text { Left atrial indexed volume }>34 \mathrm{~mL} / \mathrm{m}^{2}, \\
\mathrm{n}(\%)\end{array}$ & $20 / 56(36 \%)$ & $15 / 35(43 \%)$ & $5 / 21(24 \%)$ & 0.150 & $19 / 49(39 \%)$ & $1 / 7(14 \%)$ & 0.108 \\
\hline Septal thickness $(\mathrm{mm})^{\mathrm{a}}$ & $10.7 \pm 1.9$ & $10.31 \pm 1.94$ & $11.35 \pm 1.76$ & 0.051 & $10.67 \pm 1.95$ & $10.71 \pm 1.89$ & 0.959 \\
\hline $\begin{array}{l}\text { Left ventricular end diastolic diameter } \\
(\mathrm{mm})^{\mathrm{a}}\end{array}$ & $43.8 \pm 4.2$ & $44.33 \pm 4.30$ & $42.75 \pm 3.92$ & 0.178 & $44.10 \pm 4.23$ & $41.43 \pm 3.31$ & 0.116 \\
\hline Indexed left ventricular mass $\left(\mathrm{g} / \mathrm{m}^{2}\right)^{\mathrm{a}}$ & $85.6 \pm 24.2$ & $83.6 \pm 24.6$ & $89.1 \pm 23.6$ & 0.417 & $86.1 \pm 24.2$ & $82.1 \pm 25.4$ & 0.691 \\
\hline Relative wall thickness ${ }^{\mathrm{a}}$ & $0.45 \pm 0.09$ & $0.43 \pm 0.10$ & $0.48 \pm 0.07$ & 0.021 & $0.44 \pm 0.10$ & $0.47 \pm 0.05$ & 0.556 \\
\hline \multicolumn{8}{|l|}{ Left ventricular geometry } \\
\hline Normal & $31(54 \%)$ & $23(64 \%)$ & $8(36 \%)$ & 0.181 & $27(54 \%)$ & $4(50 \%)$ & 0.963 \\
\hline Concentric remodelling & $15(26 \%)$ & $7(19 \%)$ & $8(36 \%)$ & & $13(26 \%)$ & $2(25 \%)$ & \\
\hline Concentric hypertrophy & $10(17 \%)$ & $6(17 \%)$ & $4(19 \%)$ & & $9(18 \%)$ & $1(12.5 \%)$ & \\
\hline Eccentric hypertrophy & $0(0 \%)$ & $0(0 \%)$ & $0(0 \%)$ & & $0(0 \%)$ & $0(0 \%)$ & \\
\hline Not evaluable & $2(3 \%)$ & $0(0 \%)$ & $2(9 \%)$ & & $1(2 \%)$ & $1(12.5 \%)$ & \\
\hline Ejection fraction $(\%)^{\mathrm{a}}$ & $64.41 \pm 7.55$ & $63.31 \pm 8.94$ & $66.29 \pm 3.73$ & 0.086 & $63.90 \pm 7.92$ & $67.50 \pm 3.63$ & 0.214 \\
\hline Reduced ejection fraction, $n(\%)$ & 4/57 (7) & $4 / 36(11)$ & $0 / 21(0)$ & 0.285 & $4 / 49(8)$ & $0 / 8(0)$ & 1.000 \\
\hline $\mathrm{E} / \mathrm{A}_{\text {ratio }}{ }^{\mathrm{a}}$ & $1.08 \pm 0.48$ & $1.05 \pm 0.37$ & $1.13 \pm 0.63$ & 0.606 & $1.11 \pm 0.49$ & $0.88 \pm 0.30$ & 0.227 \\
\hline Lateral E/E' ratio ${ }^{\mathrm{a}}$ & $6.68 \pm 2.16$ & $6.64 \pm 2.07$ & $6.91 \pm 2.42$ & 0.695 & $6.46 \pm 2.1$ & $7.86 \pm 2.26$ & 0.116 \\
\hline Septal E/E' ratio ${ }^{\mathrm{a}}$ & $8.85 \pm 2.75$ & $8.05 \pm 2.04$ & $10.02 \pm 3.40$ & 0.064 & $8.70 \pm 2.86$ & $9.64 \pm 2.12$ & 0.449 \\
\hline E deceleration time, $\mathrm{msec}^{\mathrm{a}}$ & $187.25 \pm 50.81$ & $195.53 \pm 49.23$ & $174.06 \pm 51.72$ & 0.139 & $186.04 \pm 48.07$ & $195.00 \pm 70.11$ & 0.669 \\
\hline \multicolumn{8}{|l|}{ Diastolic function, $n$} \\
\hline Normal & $38(66 \%)$ & $24(67 \%)$ & $14(63 \%)$ & 0.709 & $32(64 \%)$ & $6(75 \%)$ & 1.000 \\
\hline Grade I dysfunction & $10(17 \%)$ & $7(19 \%)$ & $3(14 \%)$ & & $9(18 \%)$ & $1(12.5 \%)$ & \\
\hline Grade II dysfunction & $1(2 \%)$ & $1(3 \%)$ & $0(0 \%)$ & & $1(2 \%)$ & $0(0 \%)$ & \\
\hline Grade III dysfunction & $1(2 \%)$ & $0(0 \%)$ & $1(5 \%)$ & & $1(2 \%)$ & $0(0 \%)$ & \\
\hline Not evaluable & $8(13 \%)$ & $4(11 \%)$ & $4(18 \%)$ & & $7(14 \%)$ & $1(12.5 \%)$ & \\
\hline Right atrial volume $(\mathrm{mL})^{\mathrm{a}}$ & $46.60 \pm 23.48$ & $46.86 \pm 23.61$ & $46.11 \pm 23.91$ & 0.914 & $47.98 \pm 24.17$ & $33.40 \pm 8.05$ & 0.189 \\
\hline Right ventricular basal diameter $(\mathrm{mm})^{\mathrm{a}}$ & $36.58 \pm 6.14$ & $36.69 \pm 6.25$ & $36.40 \pm 6.09$ & 0.870 & $37.02 \pm 6.16$ & $33.57 \pm 5.50$ & 0.167 \\
\hline Ventricular basal diameters right/left ratio ${ }^{a}$ & $0.81 \pm 0.11$ & $0.80 \pm 0.12$ & $0.84 \pm 0.10$ & 0.160 & $0.81 \pm 0.12$ & $0.81 \pm 0.07$ & 0.998 \\
\hline Fractional area change $(\%)^{\mathrm{a}}$ & $47.57 \pm 6.84$ & $47.71 \pm 7.38$ & $47.32 \pm 5.91$ & 0.840 & $48.17 \pm 6.88$ & $42.83 \pm 4.54$ & 0.071 \\
\hline $\begin{array}{l}\text { Tricuspid annular plane systolic excursion } \\
(\mathrm{mm})^{\mathrm{a}}\end{array}$ & $22.94 \pm 3.37$ & $23.06 \pm 3.51$ & $22.74 \pm 3.21$ & 0.742 & $23.11 \pm 3.49$ & $21.67 \pm 2.07$ & 0.329 \\
\hline Right ventricular $S^{\prime}(\mathrm{cm} / \mathrm{sec})^{\mathrm{a}}$ & $0.14 \pm 0.03$ & $0.13 \pm 0.03$ & $0.15 \pm 0.02$ & 0.078 & $0.14 \pm 0.03$ & $0.16 \pm 0.02$ & 0.062 \\
\hline $\begin{array}{l}\text { Right ventricular systolic dysfunction, } n \\
(\%)\end{array}$ & $1 / 55(2)$ & $1 / 35(3)$ & $0 / 15(0)$ & 1.000 & $1 / 47(2)$ & $0 / 7(0)$ & 1.000 \\
\hline $\begin{array}{l}\text { Inferior vena cava espiratory diameter } \\
(\mathrm{mm})^{\mathrm{a}}\end{array}$ & $14.5 \pm 4.2$ & $13.96 \pm 4.27$ & $15.40 \pm 4.03$ & 0.298 & $14.29 \pm 4.16$ & $16.00 \pm 4.53$ & 0.399 \\
\hline Inferior vena cava collapsibility $(\%)^{\mathrm{a}}$ & $44.8 \pm 13.0$ & $45.23 \pm 14.97$ & $44.07 \pm 9.99$ & 0.795 & $45.13 \pm 13.69$ & $42.40 \pm 8.29$ & 0.670 \\
\hline $\begin{array}{l}\text { Pulmonary artery systolic pressure (mm } \\
\mathrm{Hg})^{\mathrm{a}}\end{array}$ & $27.9 \pm 5.09$ & $26.24 \pm 4.34$ & $30.67 \pm 5.16$ & 0.006 & $27.62 \pm 5.02$ & $31.33 \pm 5.69$ & 0.229 \\
\hline $\begin{array}{l}\text { Pulmonary artery systolic pressure }>35 \\
\mathrm{mmHg}, n(\%)\end{array}$ & $4 / 40(10)$ & $1 / 25(4)$ & $3 / 15(20)$ & 0.139 & $3 / 37(8)$ & 1/3 (33) & 0.161 \\
\hline Pericardial effusion, $n(\%)$ & $0 / 58(0)$ & $0 / 36(0)$ & $0 / 22(0)$ & NS & $0 / 48(0)$ & $0 / 8(0)$ & NS \\
\hline
\end{tabular}

${ }^{\mathrm{a}}$ Data reported as mean $\pm \mathrm{SD}$ 
to a higher prevalence of concentric remodeling $(0.48 \pm 0.07$ vs $0.43 \pm 0.10, p=0.021$ ).

In addition, we found that IL- 6 and D-dimer positivity was associated with higher values of sPAP $(24.6 \pm 2.3 \mathrm{vs}$ $28.9 \pm 5.0 \mathrm{mmHg}, p=0.003$, and $26.1 \pm 4.3$ vs $29.3 \pm 5.3$, $p=0.048$, respectively). No other significant correlations between biomarkers and principal LV and RV function parameters were observed.

Finally, we did not register any COVID-19 infections among the five operators directly involved in echocardiography acquisition. In particular, we did not record any symptomatic infection during the study period, nor any possible asymptomatic infection at serological screening performed at the end of the study.

\section{Discussion}

COVID-19, in particular in the severe form, is a complex disease characterized by systemic involvement, in which the temporal cascade and the effective role of single organ damage is still poorly understood.

Our prospective study showed the absence of early significant major myocardial dysfunction in a cohort of patients admitted to a middle intensity care department. In particular, in both mild/moderate and severe pneumonia patients we did not find new onset LV dysfunction (with the exception of the patient with acute coronary syndrome evidence) or significant RV impairment, showing just a mild -but significant- increase in SPAP in the more compromised group. These observations are apparently in contrast with previous data $[17,18]$. We think that our results have to be interpreted taking into account some methodological differences with respect to previous studies. Argulian et al. described RV dilation in about $30 \%$ of COVID patients [17]. Differently from our study, in which echocardiography was performed in consecutive COVID pneumonia patients, these Authors analyzed the results of "clinically indicated" echocardiographic examinations, thus considering a selected population. In that study, roughly one third of the patients were invasively ventilated, while this subset of patient was not included in our population, as we focused on early cardiac dysfunction before the eventual onset of mechanical ventilation. A high percentage of ICU, mechanically ventilated patients was also present in a recent study in which a frequent LV systolic dysfunction was described [18]. The absence of new onset LV dysfunction in our cohort is in line with data reported by Yuman Li et al., where a smaller percentage of intubated patients was enrolled [19]. On the other hand, the same Authors reported a positive correlation between lower values of RV systolic function parameters and worse prognosis, although the considered cut-off values for $\mathrm{RV}$ parameters were largely within the normal range even in the adverse event group [19]. It is important to underline that, differently from previous studies, we assessed an early evaluation of cardiac involvement, performing echocardiographic examinations within $72 \mathrm{~h}$ from admission. The absence of significant pathological findings even in the most severely compromised patients, seem not to indicate the heart as one of the target organs at least in this phase of the illness. Nevertheless, we cannot exclude that subtle functional modification could have some relevance. In these perspectives, even cardiac parameters in the lower range of normality shown in some studies could represent the signal of a reduced functional reserve, possibly playing a role in the later complex development of the systemic response to the virus-triggered cascade. The detection of a higher SPAP in our more severely affected patients could be part of this picture, in particular reflecting an early pulmonary vascular perturbation, not unexpected in a condition of overt lung involvement. Of notice, sPAP elevation after 2 months from discharge was shown in up to $8 \%$ of patients with moderate COVID disease [20].

Elevated serum hs-TnT levels were observed in one third of our cohort, with a significant difference between survivors and non-survivors, in line with previous reports [3, 4, 21]. In our population this biomarker was related to adverse events in the absence of an association with electrocardiographic or echocardiographic changes. This observation could confirm the hypothesis that troponin elevation mainly represents a warning sign of systemic involvement rather than a proper marker of cardiac injury [4, 22]. On the other hand, a more specific role of increased troponin as a sign of subclinical cardiac dysfunction has to be kept in consideration.

Our results confirm the important association between outcomes and clinical parameters like age, history of hypertension, P/F and biomarkers [23]. Interestingly, echocardiography parameters potentially related to preexisting hypertensive cardiomyopathy, like relative wall thickness and concentric remodeling, were more frequent in severely affected patients.

IL-6 was significantly related to lung involvement and death, confirming the role of severe inflammation in the disease course, according to previous literature [24]. Similarly, D-Dimer elevation was associated with disease severity and prognosis. This association could reflect the role of hypercoagulability that has extensively been highlighted in COVID disease [25]. Moreover, the IL-6 and D-dimer significant correlation with SPAP value may reflect the detrimental role of cytokinin storm in heart-lung interaction.

The limit of our study is related to the restricted number of enrolled patients, which did not allow us to further investigate the prognostic characteristics within the severe pneumonia group. Moreover, the enrollment of patients in a middle intensity department excludes patients with critical illness at presentation. Nevertheless, our event rate in the 
severe form of the disease was fairly high (36\%), reflecting a severe subsequent evolution in a part of our population, in line with the described natural history of COVID pneumonia. The strength of our study was the prospective and consecutive enrollment of patients that avoided the possible selection bias potentially affecting previous studies that enrolled patients with echocardiography performed for cardiological concerns. Moreover, we focused on the early phase of the disease, before eventual overt hemodynamic compromise and mechanical ventilation onset.

In conclusion, a mildly increased SPAP in severe COVID19 pneumonia patients with respect to those with milder disease was the only significant finding at early echocardiographic examination. We did not find early new onset major COVID-related myocardial dysfunction, as evaluated by routinely performed echocardiographic parameters, in our cohort of consecutive patients hospitalized in a medium intensity care department. Echocardiography remains an irreplaceable tool to detect major cardiac complications, like acute coronary syndrome or myocarditis, and to assess preexisting cardiovascular impairment, in particular in comorbid populations [26]. Moreover, the possibility to perform bedside examination without evidence of an increased risk for properly protected operators, make echocardiography suitable for the non-invasive hemodynamic monitoring, without displacing COVID infected patients. Future studies with repeated measurements, in particular during the detrimental phase of the disease, and post-discharge follow-up are needed to deepen the knowledge regarding the timing and possible complementary role of cardiac function reserve alterations in the evolution of a complex systemic disorder in which respiratory involvement appears as the main character, at least in non-ICU patients.

Acknowledgements Dr. Martina Perego, Dr. Emanuele Salvi and Dr. Thomas Teatini, for the contribution in managing patients and study database.

\section{Declarations}

Conflict of interest The authors declare that they have no conflict of interest.

Human and animal rights The study protocol complied with the Declaration of Helsinki.

Informed consent The study was approved by the local Ethics Committee and informed consent was obtained.

\section{References}

1. Zhu N, Zhang D, Wu W et al (2020) China novel coronavirus investigating and research team. N Engl J Med 382:727-733. https://doi.org/10.1017/CBO9781107415324.004
2. Clerkin KJ, Fried JA, Raikhelkar J et al (2020) Coronavirus disease 2019 (COVID-19) and cardiovascular disease. Circulation. https://doi.org/10.1161/CIRCULATIONAHA.120.046941

3. Shi S, Qin M, Shen B et al (2020) Association of cardiac injury with mortality in hospitalized patients with COVID-19 in Wuhan, China. JAMA Cardiol. https://doi.org/10.1001/jamac ardio.2020.0950

4. Ghio S, Baldi E, Vicentini A et al (2020) Cardiac involvement at presentation in patients hospitalized with COVID-19 and their outcome in a tertiary referral hospital in Northern Italy. Intern Emerg Med 15:1457-1465. https://doi.org/10.1007/ s11739-020-02493-y

5. Cipriani A, Capone F, Donato F et al (2020) Cardiac injury and mortality in patients with coronavirus disease 2019 (COVID19): insights from a mediation analysis. Intern Emerg Med. https://doi.org/10.1007/s11739-020-02495-w

6. Inciardi RM, Lupi L, Zaccone G et al (2020) Cardiac Involvement in a patient with coronavirus disease 2019 (COVID-19). JAMA Cardiol 2019:1-6. https://doi.org/10.1001/jamacardio. 2020.1096

7. Doyen D, Moceri P, Ducreux D et al (2020) Myocarditis in a patient with COVID-19: a cause of raised troponin and ECG changes. Lancet. https://doi.org/10.1016/S0140-6736(20) 30912-0

8. Alemzadeh-Ansari M (2020) Coronavirus disease 2019 (COVID19) and cardiovascular events. Res Cardiovasc Med 9:1. https:// doi.org/10.4103/rcm.rcm_9_20

9. Ibrahim A, Walid H, Nadia $\mathrm{S}$ et al (2019) The prognostic significance of elevated cardiac troponin in non-cardiac medical disorders. Pilot study. Int J Clin Cardiol. https://doi.org/10.23937/ 2378-2951/1410136

10. Barman HA, Atici A, Tekin EA et al (2020) Echocardiographic features of patients with COVID-19 infection: a cross-sectional study. Int J Cardiovasc Imaging. https://doi.org/10.1007/ s10554-020-02051-9

11. Cogliati C, Ceriani E, Brambilla AM (2020) When internal and emergency medicine speak to each other : organization in the time of COVID. Intern Emerg Med. https://doi.org/10.1007/ s11739-020-02380-6

12. Ryan T, Berlacher K, Lindner JR et al (2015) COCATS 4 task force 5: training in echocardiography. J Am Coll Cardiol 65:17861799. https://doi.org/10.1016/j.jacc.2015.03.035

13. Siegel JD, Rhinehart E, Jackson M et al (2019) 2007 guideline for isolation precautions: preventing transmission of infectious agents in healthcare settings. https://www.cdc.gov/infectioncontrol/guide lines/isolation/index.html. Accessed July 2019

14. Lang RM, Badano LP, Mor-Avi V et al (2015) Recommendations for cardiac chamber quantification by echocardiography in adults: an update from the American Society of Echocardiography and the European Association of Cardiovascular Imaging. Eur Heart J Cardiovasc Imaging 16:233-270. https://doi.org/10.1093/ehjci/ jev014

15. Rudski LG, Lai WW, Afilalo J et al (2010) Guidelines for the echocardiographic assessment of the right heart in adults: a report from the american society of echocardiography. J Am Soc Echocardiogr. https://doi.org/10.1016/j.echo.2010.05.010

16. Wu Z, McGoogan JM (2020) Characteristics of and important lessons from the coronavirus disease 2019 (COVID-19) outbreak in china. JAMA. https://doi.org/10.1001/jama.2020.2648

17. Argulian E, Sud K, Vogel B et al (2020) Right ventricular dilation in hospitalized patients with COVID-19 infection. JACC Cardiovasc Imaging. https://doi.org/10.1016/j.jcmg.2020.05.010

18. Churchill TW, Bertrand PB, Bernard S et al (2020) Echocardiographic features of COVID-19 illness and association with cardiac biomarkers. J Am Soc Echocardiogr. https://doi.org/10.1016/j. echo.2020.05.028 
19. Li Y, Li H, Zhu S et al (2020) Prognostic value of right ventricular longitudinal strain in patients with COVID-19. JACC Cardiovasc Imaging. https://doi.org/10.1016/j.jcmg.2020.04.014

20. Tudoran C, Tudoran M, Lazureanu VE et al (2021) Evidence of pulmonary hypertension after SARS-CoV-2 infection in subjects without previous significant cardiovascular pathology. J Clin Med. https://doi.org/10.3390/jcm10020199

21. Lombardi CM, Carubelli V, Iorio A et al (2020) Association of troponin levels with mortality in Italian patients hospitalized with coronavirus disease 2019. JAMA Cardiol. https://doi.org/10.1001/ jamacardio.2020.3538

22. Deng Q, Hu B, Zhang Y et al (2020) Suspected myocardial injury in patients with COVID-19: evidence from front-line clinical observation in Wuhan, China. Int J Cardiol. https://doi.org/10. 1016/j.ijcard.2020.03.087

23. Wynants L, Van Calster B, Bonten MMJ et al (2020) Prediction models for diagnosis and prognosis of covid-19 infection: systematic review and critical appraisal. BMJ. https://doi.org/10. 1136/bmj.m1328

24. Herold T, Jurinovic V, Arnreich C et al (2020) Level of IL-6 predicts respiratory failure in hospitalized symptomatic COVID-19 patients. MedRxiv. https://doi.org/10.1101/2020.04.01.20047381

25. Panigada M, Bottino N, Tagliabue P et al (2020) Hypercoagulability of COVID-19 patients in intensive care unit. A report of thromboelastography findings and other parameters of hemostasis. J Thromb Haemost. https://doi.org/10.1111/jth.14850

26. Cameli M, Pastore MC, Soliman Aboumarie H et al (2020) Usefulness of echocardiography to detect cardiac involvement in COVID-19 patients. Echocardiography. https://doi.org/10.1111/ echo. 14779

Publisher's Note Springer Nature remains neutral with regard to jurisdictional claims in published maps and institutional affiliations. 